\title{
STEP to estimate cardiovascular events by home blood pressure in the era of digital hypertension
}

\author{
Kazuomi Kario ${ }^{1} \cdot$ Atsushi Sakima ${ }^{2,3} \cdot$ Yusuke Ohya $^{2}$
}

Received: 10 September 2021 / Revised: 14 September 2021 / Accepted: 15 September 2021 / Published online: 17 October 2021

(c) The Japanese Society of Hypertension 2021

Recently, the STEP trial (Strategy of Blood Pressure Intervention in the Elderly Hypertensive Patients) clearly demonstrated that intensive treatment with a systolic blood pressure (BP) target of $110-130 \mathrm{mmHg}$ resulted in a lower incidence of cardiovascular events than standard treatment with a systolic BP target of $130-150 \mathrm{mmHg}$ in older patients with hypertension [1]. The target BP in older hypertensive patients is controversial and varies among the different guidelines for hypertension management [2-4]. In the STEP trial, adjustment of antihypertensive medication was based on office BP, and a successful adjustment was considered to be one that resulted in a difference of $9.3 \mathrm{mmHg}$ between the intensive treatment and standard treatment groups. In the STEP trial, it was clinically important that the home BP monitoring be used to evaluate the BP control [5]. The average difference in morning home systolic BP between the intensive and standard management groups was around $7.5 \mathrm{mmHg}$. As a result, all the cardiovascular events, including stroke, coronary artery disease, and heart failure, were markedly suppressed in the intensive BP management group. The benefit to patients with heart failure was greatest, with a hazard ratio of 0.27 .

\section{Masked uncontrolled morning hypertension}

Compared with office BP, home BP exhibited a greater seasonal variation in the STEP study paper, as shown in its

Kazuomi Kario

kkario@jichi.ac.jp

1 Division of Cardiovascular Medicine, Department of Medicine, Jichi Medical University School of Medicine,

Tochigi, Shimotsuke, Japan

2 Department of Cardiovascular Medicine, Nephrology and Neurology, Graduate School of Medicine, University of the Ryukyus, Okinawa, Japan

3 Health Administration Center, University of the Ryukyus, Okinawa, Japan supplemental materials. Over the course of the follow-up, while office systolic BP remained well-controlled at $<140$ $\mathrm{mmHg}$, the morning home systolic BP increased to $>135$ $\mathrm{mmHg}$ in the standard group. Thus, the prevalence of masked morning hypertension seems to have increased in the standard treatment group. On the other hand, patients in the intensive control groups exhibited sustained control of both office and morning home systolic BP, indicating that there was no increase in masked uncontrolled hypertension throughout the study. In our prospective studies-i.e., the J-HOP (Japan Morning Surge-Home Blood Pressure) study and HONEST (Home Blood Pressure Measurement With Olmesartan Naïve Patients to Establish Standard Target Blood Pressure (HONEST) study—both of which were general practitioner-based cohort studies enrolling a large number of patients on antihypertensive medication, masked hypertension, and poorly-controlled morning hypertension were associated with cardiovascular events [6-9]. Because hypertension management in the STEP study was based on adjustment of office BP, rather than home BP, the intensive treatment targeting office systolic $\mathrm{BP}<130 \mathrm{mmHg}$ may be sustained to achieve well-controlled morning home BP. In contrast, a standard treatment based on office BP seems insufficient to achieve good control of morning BP along with longer follow-up. Thus, the STEP study indicates the importance of controlling uncontrolled morning hypertension, which is frequently observed in patients receiving standard management in the current clinical practice.

\section{Morning home BP-estimated cardiovascular risk}

The reduction in cardiovascular event risk by lowering morning home BP was estimated using data from the J-HOP study and the STEP study (Table 1). Based on this estimation, we calculated the cardiovascular event risks separately for stroke, coronary artery disease, and heart failure for the morning SBP lowering effect found in recent clinical 
Table 1 Morning home blood pressure-estimated cardiovascular risk by J-HOP and STEP

\begin{tabular}{lrrrr}
\hline Difference in morning SBP & \multicolumn{4}{c}{ Difference in cardiovascular event (\%) } \\
\cline { 2 - 5 } & Stroke & CAD & \multicolumn{1}{l}{ HF } & Total CVD \\
\hline J-HOP & & & & \\
$-2.5 \mathrm{mmHg}$ & -7.0 & 0.7 & -5.2 & -3.5 \\
$-5.0 \mathrm{mmHg}$ & -13.4 & 1.4 & -10.2 & -6.9 \\
$-7.5 \mathrm{mmHg}$ & -19.4 & 2.1 & -14.9 & -10.2 \\
$-10.0 \mathrm{mmHg}$ & -25.0 & 2.9 & -19.4 & -13.3 \\
$-15.0 \mathrm{mmHg}$ & -35.1 & 4.3 & -27.6 & -19.3 \\
$-20.0 \mathrm{mmHg}$ & -43.8 & 5.8 & -35.0 & -24.9 \\
$\mathrm{STEP}$ & & & & \\
$-2.5 \mathrm{mmHg}$ & -12.5 & -12.5 & -35.4 & -9.5 \\
$-5.0 \mathrm{mmHg}$ & -23.4 & -23.4 & -58.2 & -18.2 \\
$-7.5 \mathrm{mmHg}$ & -33.0 & -33.0 & -73.0 & -26.0 \\
$-10.0 \mathrm{mmHg}$ & -41.4 & -41.4 & -82.5 & -33.1 \\
$-12.5 \mathrm{mmHg}$ & -48.7 & -48.7 & -88.7 & -39.5 \\
$-15.0 \mathrm{mmHg}$ & -55.1 & -55.1 & -92.7 & -45.2 \\
$-20.0 \mathrm{mmHg}$ & -65.6 & -65.6 & -97.0 & -55.2 \\
\hline
\end{tabular}

The J-HOP (Japan Morning Surge-Home Blood Pressure: a practitioner-based observation study) study; STEP (Strategy of Blood Pressure Intervention in the Elderly Hypertensive Patients: an intervention trial on older hypertensive patients) trial

studies, as shown in Table 2. For antihypertensive medication, the time period with the weakest treatment effect is in the morning before taking the morning pill, because of the shorter BP-lowering effect. Even when we used wearable BP monitoring devices, morning systolic BP measured based on the guideline-recommended measurements was most closely associated with hypertensive organ damage in medicated hypertensives [10].

There are several approaches for controlling morning BP other than antihypertensive medication. New approaches such as digital therapeutics and device treatment, have begun to be introduced for the management of hypertension. In our recent randomized controlled trial (RCT) of digital therapeutics, the HERB Digital Hypertension 1 (HERB DH1) pivotal study, which facilitates individual lifestyle modifications on six guideline-recommended components (decrease salt intake, body weight control, exercise, improving sleep condition, stress coping, and reducing alcohol intake), demonstrated an effective BP-lowering effect on both $24 \mathrm{~h}$ ambulatory BP and morning home systolic BP [11]. Evaluation of salt intake and restriction is crucially important for the non-pharmacological treatment of hypertension [12]. The morning SBP difference found in the HERB DH1 pivotal study $(4.3 \mathrm{mmHg}$ reduction in the digital therapeutic group vs. control group) have sufficient impact on the reduction in the cardiovascular events risk (Table 2).

For patients with resistant hypertension, renal denervation is another approach. Four sham-controlled trials of catheter-
Table 2 Cardiovascular event risk reductions estimated by using the J-HOP or STEP data

\begin{tabular}{|c|c|c|c|c|}
\hline \multirow{2}{*}{ Difference in morning SBP } & \multicolumn{4}{|c|}{ Difference in cardiovascular event $(\%)$} \\
\hline & Stroke & CAD & $\mathrm{HF}$ & Total CVD \\
\hline \multicolumn{5}{|l|}{ Clinical trials } \\
\hline \multicolumn{5}{|l|}{ HERB DH-1 $\left(-4.3 \mathrm{mmHg}^{\mathrm{a}}\right)$} \\
\hline Estimated by J-HOP & -11.7 & 1.2 & -8.8 & -6.0 \\
\hline Estimated by STEP & -20.5 & -20.5 & -52.8 & -15.9 \\
\hline \multicolumn{5}{|l|}{ RDN-Meta $\left(-3.7 \mathrm{mmHg}^{\mathrm{b}}\right)$} \\
\hline Estimated by J-HOP & -10.1 & 1.1 & -7.7 & -5.2 \\
\hline Estimated by STEP & -17.9 & -17.9 & -47.6 & -13.8 \\
\hline \multicolumn{5}{|l|}{ REQUIRE $\left(-5.4 \mathrm{mmHg}^{\mathrm{c}}\right)$} \\
\hline Estimated by J-HOP & -14.4 & 1.5 & -11.0 & -7.4 \\
\hline Estimated by STEP & -25.0 & -25.0 & -61.0 & -19.5 \\
\hline \multicolumn{5}{|l|}{ REQUIRE $\left(-1.8 \mathrm{mmHg}^{\mathrm{d}}\right)$} \\
\hline Estimated by J-HOP & -5.1 & 0.5 & -3.8 & -2.5 \\
\hline Estimated by STEP & -9.2 & -9.2 & -27.0 & -7.0 \\
\hline \multicolumn{5}{|l|}{ HTN-J $\left(-5.6 \mathrm{mmHg}^{\mathrm{e}}\right)$} \\
\hline Estimated by J-HOP & -14.9 & 1.6 & -11.4 & -7.7 \\
\hline Estimated by STEP & -25.8 & -25.8 & -62.4 & -20.1 \\
\hline
\end{tabular}

The J-HOP (Japan Morning Surge-Home Blood Pressure: a practitioner-based observation study) study (Hoshide et al. [6]); STEP (Strategy of Blood Pressure Intervention in the Elderly Hypertensive Patients: an intervention trial on older hypertensive patients) trial (Zhang et al. [1]); HERB DH-1 (HERB Digital Hypertension-1) pivotal study (Kario et al. [11]); and REQUIRE (REnal Denervation on Quality of 24-h BP Control by Ultrasound In REsistant Hypertension) trial (Kario et al. Circ J. 2015; 79:1222-1229) were compared

$C A D$ coronary artery disease, $C V D$ cardiovascular disease, $H F$ heart failure, $S B P$ systolic blood pressure

${ }^{\text {a }}$ Difference in the morning home SBP between the digital therapeutic and control groups

${ }^{\mathrm{b}}$ Difference in the home SBP between the renal denervation and sham groups in the meta-analysis (Ogoyama et al. [13]) of 4 sham-controlled trials (SYMPLICITY HTN-3, RADIANCE-HTN SOLO, RADIANCE-HTN TRIO, and REQUIRE)

${ }^{\mathrm{c}}$ Difference in the 1 month

${ }^{\mathrm{d}}$ Difference in the 3 months home SBP reduction between the renal denervation and sham groups.

${ }^{\mathrm{e}}$ Difference in the home SBP $(-5.64 \mathrm{mmHg})$ between the renal denervation $(n=22)$ and control $(n=19)$ groups in the HTN-J study $(-8.00$ vs. $-2.36 \mathrm{mmHg}, p=0.205)$ [Kario et al. Circ J. 2015; 79:1222-1229]

based renal denervation were included in a recent metaanalysis on the effects of renal sympathetic denervation on blood pressure [13]. The REQUIRE trial, a sham-controlled RCT using an ultrasound device system, did not achieve the primary endpoint of $24 \mathrm{~h}$ systolic BP at 3 months, compared with the sham-control [14]. Heightened awareness of high BP may lead to changes in patient adherence behavior or lifestyle modifications after randomization that could be more prevalent in the sham group if the procedure is effective [14]. 
In the REQUIRE trial, significant difference was found in home BP at 1 month between the renal denervation and sham groups, but this difference disappeared at 3 months according to the progressive reduction in the sham group. The benefit of these new treatments is that they achieve a sustained BPlowering effect throughout $24 \mathrm{~h}$, including the night-time and morning periods. The significant home BP reduction seems to lead to the potential reduction of cardiovascular disease (Table 2). As an adjunct or alternative to medication therapy, digital therapeutics of non-pharmacological treatment, and/or renal denervation could be a useful approach in the future. The shared decision-making process based on the patient preference $[15,16]$ and doctor's opinion will facilitate an increase in adherence and decrease in clinical inertia as part of effective $24 \mathrm{~h}$ BP management, resulting in "zero" cardiovascular events.

\section{Digital hypertension management}

One of the unique aspects of the STEP trial is that all the study patients and doctors used the smartphone-based Hypertension Doctor App platform. The App aims to help patients adhere to medication and monitor BPs by several modules, including a link with medication records and antihypertensive treatment plan, graphic data of HBP during the follow-up, interactive communications between patients and physicians, and cardiovascular health education. The study subjects were randomized into the smartphone-based Hypertension Doctor App platform for BP management (with interactive communication between the Doctor Portal and Patient Portal) and the usual care group (without interaction between the Doctor portal and Patient portal). Digital hypertension management seems to contribute to sustained reductions in office and home BP [17], and successful achievement of the separation of the different BP levels on treatment in the intensive management and standard management groups.

In conclusion, STEP indicates the future direction of hypertension management-namely, digital hypertension management with an initial focus on morning BP.

\section{Compliance with ethical standards}

Conflict of interest K.K. has received consulting/lecture fees from JIMRO, A\&D, CureApp and Daiichi Sankyo and research funding from Omron Healthcare and Fukuda Denshi, Scholarship funding from Daiichi Sankyo. None of the other authors have any conflicts of interest to disclose.

Publisher's note Springer Nature remains neutral with regard to jurisdictional claims in published maps and institutional affiliations.

\section{References}

1. Zhang W, Zhang S, Deng Y, Wu S, Ren J, Sun G, et al. For the STEP Study Group. Trial of intensive blood-pressure control in older patients with hypertension. N Engl J Med. 2021. https://doi. org/10.1056/NEJMoa2111437.

2. Williams B, Mancia G, Spiering W, Agabiti Rosei E, Azizi M, Burnier M, et al. ESC Scientific Document Group. 2018 ESC/ ESH Guidelines for the management of arterial hypertension. Eur Heart J. 2018;39:3021-104.

3. Whelton PK, Carey RM, Aronow WS, Casey DE Jr, Collins KJ, Dennison Himmelfarb C, et al. 2017 ACC/AHA/AAPA/ABC/ ACPM/AGS/APhA/ASH/ASPC/NMA/PCNA Guideline for the prevention, detection, evaluation, and management of high blood pressure in adults: executive summary: a report of the American College of Cardiology/American Heart Association Task Force on Clinical Practice Guidelines. Hypertension. 2018;71:1269-324.

4. Umemura S, Arima H, Arima S, Asayama K, Dohi Y, Hirooka Y, et al. The Japanese Society of Hypertension Guidelines for the management of hypertension (JSH 2019). Hypertens Res. 2019;42:1235-481.

5. Kario K, Shimbo D, Hoshide S, Wang JG, Asayama K, Ohkubo $\mathrm{T}$, et al. The emergence of home blood pressure-guided management of hypertension based on global evidence. Hypertension. 2019;74:229-36.

6. Hoshide S, Yano Y, Haimoto H, Yamagiwa K, Uchiba K, Nagasaka S, et al. Morning and evening home blood pressure and risks of incident stroke and coronary artery disease in the Japanese General Practice Population: The Japan Morning Surge-Home Blood Pressure Study. Hypertension 2016;68:54-61.

7. Kario K, Saito I, Kushiro T, Teramukai S, Tomono Y, Okuda Y, et al. Morning Home blood pressure is a strong predictor of coronary artery disease: The HONEST Study. J Am Coll Cardiol. 2016;67:1519-27.

8. Fujiwara T, Yano Y, Hoshide S, Kanegae H, Kario K. Association of cardiovascular outcomes with masked hypertension defined by home blood pressure monitoring in a Japanese ceneral practice population. JAMA Cardiol. 2018;3:583-90.

9. Narita K, Hoshide S, Kario K. Difference between morning and evening home blood pressure and cardiovascular events: the J-HOP Study (Japan Morning Surge-Home Blood Pressure). Hypertens Res. 2021. https://doi.org/10.1038/s41440-021-00686-2.

10. Kario K, Tomitani N, Morimoto T, Kanegae H, Lacy P, Williams B. Relationship between blood pressure repeatedly measured by a wrist-cuff oscillometric wearable blood pressure monitoring device and left ventricular mass index in working hypertensive patients. Hypertens Res. 2021. https://doi.org/10.1038/s41440021-00758-3.

11. Kario K, Nomura A, Harada N, Okura A, Nakagawa K, Tanigawa $\mathrm{T}$, et al. Efficacy of a digital therapeutics system for the management of essential hypertension (HERB-DH1 pivotal) in the absence of antihypertensive medications: the multicentre, randomised, open-label trial. Eur Heart J. 2021. Online.

12. Yamazato M, Sakima A, Ishida A, Kohagura K, Matayoshi T, Tana $\mathrm{T}$, et al. Salt and potassium intake evaluated with spot urine and brief questionnaires in combination with blood pressure control status in hypertensive outpatients in a real-world setting. Hypertens Res. 2021. https://doi.org/10.1038/s41440-021-00707-0.

13. Ogoyama Y, Tada K, Abe M, Nanto S, Shibata H, Mukoyama M, et al. Effects of renal sympathetic denervation on blood pressure among hypertensive patients: a systematic review and metaanalysis of randomised sham-controlled trials. Hypertens Res. 2021;22:572-84.

14. Kario K, Yokoi Y, Okamura K, Fujihara M, Ogoyama Y, Yamamoto E, et al. Catheter-based ultrasound renal denervation in 
patients with resistant hypertension: the randomized, controlled REQUIRE trial. Hypertens Res. 2021. in press.

15. Schmieder RE, Mahfoud F, Mancia G, Azizi M, Böhm M, Dimitriadis K. Members of the ESH Working Group on DeviceBased Treatment of Hypertension et al. European Society of Hypertension position paper on renal denervation 2021. J Hypertens. 2021;39:1733-41.
16. Kario K, Kagitani H, Hayashi S, Hanamura S, Ozawa K, Kanegae H. A Japan nationwide web-based survey of patient preference for renal denervation for hypertension treatment. Hypertens Res. 2021. in press.

17. Omboni S, McManus RJ, Bosworth HB, Chappell LC, Green BB, Kario K, et al. Evidence and recommendations on the use of telemedicine for the management of arterial hypertension: an International expert position paper. Hypertension 2020;76:1368-83. 